\title{
Comparativo entre os requisitos das concessionárias de energia utilizando modelos BIM
}

\section{Compariso between the requirements of energy utilities using BIM models}

\section{Eduardo Clasen Inácio}

Universidade Paulista| São Paulo | Brasil | eduardo.clasen.inacio@gmail.com

\section{Sandra Albino Ribeiro}

Quatre Ensino Especializado | Natal | Brasil | sandra@espacoquatre.com

\section{Resumo}

As redes de distribuição subterrânea de energia elétrica vêm ganhando cada vez mais espaço nas cidades brasileiras em razão de suas vantagens. O fato de existirem diversas concessionárias, com diferentes normas, faz com que um mesmo projeto desta natureza possa apresentar características únicas. Considerando esse contexto, o presente trabalho visa desenvolver um comparativo entre três concessionárias de energia a partir da elaboração de projetos em BIM. O método adotado foi o da pesquisa descritiva e o procedimento o estudo de caso. Os resultados revelam os critérios que oneram mais o projeto e as vantagens da adoção do BIM. Entre as contribuições tem-se a difusão desse novo paradigma em projetos de infraestrutura.

Palavras-chave: Projeto de rede de distribuição subterrânea de energia elétrica. Concessionária de energia. BIM. Modelos BIM. Infraestrutura urbana.

\begin{abstract}
Underground electricity distribution networks are gaining more and more space in Brazilian cities due to their advantages. The fact that there are several concessionaires, with different standards, means that the same project of this nature can present unique characteristics. Considering this context, the present work aims to develop a comparison between three concessionaires based on the development of projects in BIM. The method adopted was that of descriptive research and the procedure was a case study. The results reveal the advantages of adopting BIM and that some criteria burden the project more. Among the contributions is the dissemination of this new paradigm in infrastructure projects
\end{abstract}

Keywords: Underground electricity distribution network project. Concessionaire. BIM. BIM models. Urban infrastructure.

Como citar:

INACIO, E. C. .; RIBEIRO, S. A. Comparativo entre os requisitos das concessionárias de energia utilizando modelos BIM. In: SIMPÓSIO BRASILEIRO DE TECNOLOGIA DA INFORMAÇÃO E COMUNICAÇÃO NA CONSTRUÇÃO, 3., 2021, Uberlândia. Anais [...]. Porto Alegre: ANTAC, 2021. p. 110. Disponível em: https://eventos.antac.org.br/index.php/sbtic/article/view/600. Acesso em: 3 ago. 2021. 


\section{INTRODUÇÃO}

As topologias aplicadas no transporte de energia elétrica são compostas por dois grupos. O primeiro é o de Rede de Distribuição Aérea (RDA) com variáveis (convencional, isolada e compacta), devido ao seu baixo custo e as facilidades na implementação é o mais difundido no Brasil. O segundo grupo é formado pela Rede de Distribuição Subterrânea (RDS) que é composto pelos tipos semienterrada e enterrada [1][2][3].

Este grupo tem ganhado notório espaço nas cidades brasileiras em virtude de suas vantagens, tais como a redução significativa das interrupções da energia elétrica, a preservação da paisagem, o aumento da segurança para a população dada a redução do risco de acidentes por ruptura de condutores e por contatos acidentais, a diminuição dos custos de manutenção com podas de árvores e deslocamento das equipes de manutenção [1][2][3]. Os benefícios gerados por essa instalação pagam ao longo do tempo o seu custo mais elevado na execução.

Os responsáveis pela distribuição de energia seguem as recomendações da Associação Brasileira de Normas Técnicas (ABNT), da Associação Brasileira de Distribuidores de Energia Elétrica (ABRADEE), da Agência Nacional de Energia Elétrica (ANEEL) e do Comitê de Distribuição (CODI). Além das suas próprias normas e resoluções técnicas que fazem com que um projeto de rede de distribuição possa apresentar características únicas e ser mais oneroso quando comparado a outra concessionária.

Paralelo ao crescimento da adoção das redes de distribuição subterrâneas, vem também aumentando a difusão e uso do BIM, acrônimo do Building Information Modelling. Isso ocorre dados aos seus benefícios vistos em todas as etapas do ciclo de vida de um empreendimento: "da concepção, passando pelo planejamento da construção, operação (que envolve uso, gestão e manutenção) e reciclagem ou demolição" [4]. Além disso, algumas referências [5] consideram o BIM como a expressão mais atual da inovação da construção civil e chave para adentrar na indústria 4.0 .

Apesar do BIM proporcionar vários ganhos, muitos dos quais já debatidos amplamente na academia, os estudos e experimentos práticos na fase de projeto para a rede de distribuição subterrâneas ainda são escassos. Ao realizar uma análise na última publicação do SBTIC, constatou-se que das 70 publicações não há nenhuma sobre esse assunto. Considerando esse contexto, o presente trabalho buscou responder de que maneira o uso de uma ferramenta de autoria BIM pode contribuir na geração de projetos de distribuição subterrânea de energia elétrica e iluminação pública. Para tal teve-se como objetivo geral desenvolver um comparativo entre três concessionárias (CELESC D, CEMIG D e CPFL ENERGIA) a partir de modelos BIM. 


\section{MÉTODO}

O método adotado foi o da pesquisa descritiva apoiando-se no estudo de caso e com abordagem qualitativa. Inicialmente, foi realizada a fundamentação teórica em BIM e em suas ferramentas de autoria aplicadas aos projetos rede de distribuição subterrânea de energia elétrica e iluminação pública. Em seguida, foram levantados e analisados os critérios normativos das concessionárias escolhidas para o estudo mais as NBR 5410:2004 [6], NBR 14039:2005 [7] e demais normas. Logo após, foram elaborados os projetos em BIM para um loteamento residencial, fictício, conforme os padrões das três concessionárias. Ao final, os resultados foram analisados e comparados a fim de identificar as diferenças entre os critérios de cada concessionárias e como a ferramenta utilizada impactava na fase de projeto.

REDE DE DISTRIBUIÇÃO SUBTERRÂNEA DE ENERGIA ELÉTRICA E BIM

Figura 1: Rede de distribuição subterrânea de energia elétrica

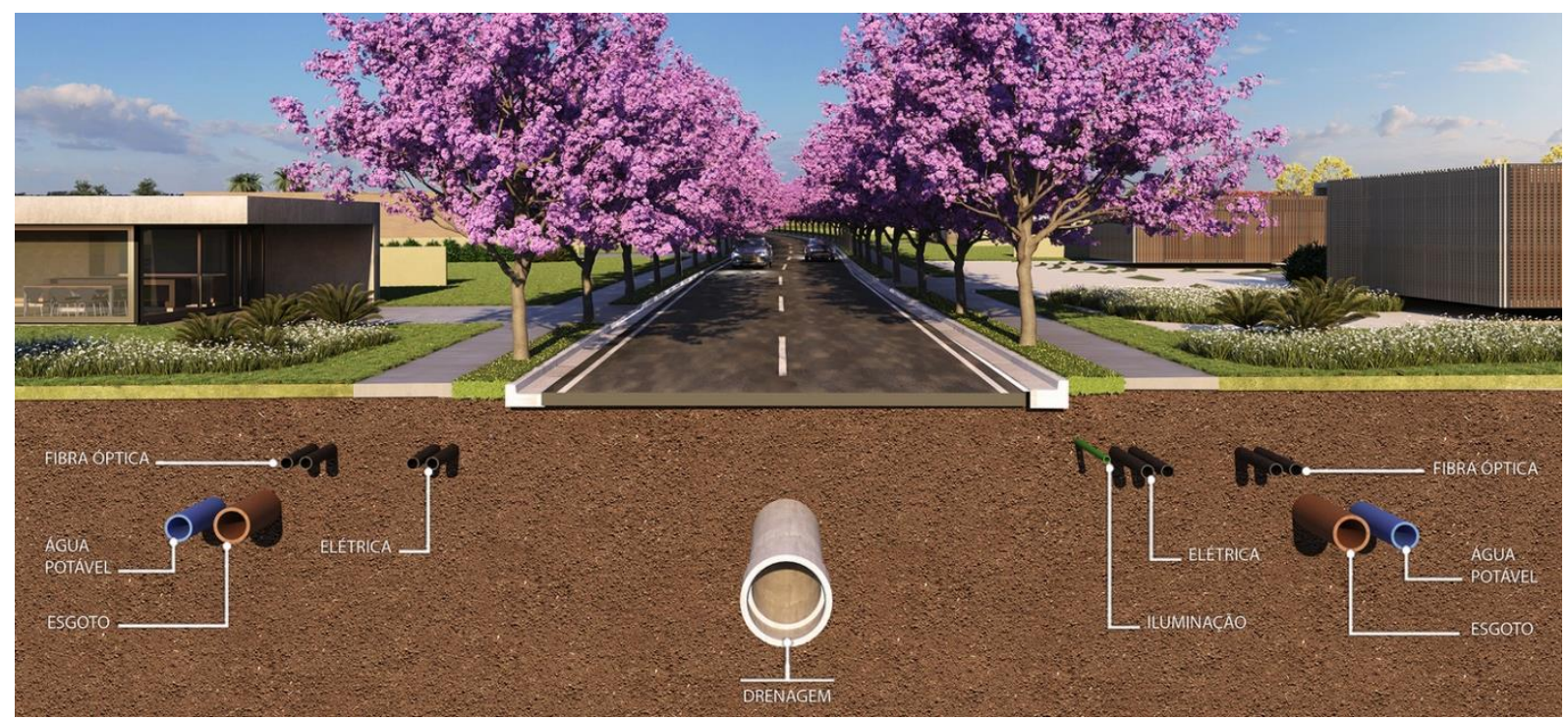

Fonte: https://cdn.douradosnews.com.br/upload/images/ext21-inforgrafico-calcadas-hr.jpg

As redes de distribuição subterrâneas de energia elétrica são denominadas pela ABNT NBR 5410:2004 [6] e NBR 14039:2005 [7] de linhas enterradas, como o próprio nome sugere, são linhas que estão localizadas abaixo do nível do solo (Figura 1). Para implantação deste sistema, é necessário que vários procedimentos sejam analisados e planejados.

Na fase do planejamento se define a configuração da rede, o levantamento e avaliação dos custos, a confiabilidade, a revitalização ou reurbanização do local e o gerenciamento correto do uso do solo, que em outras palavras, indica a necessidade de realizar o compartilhamento do subsolo e a compatibilização dos projetos (água fria, esgoto, galerias e águas pluviais, infraestrurua para semáforos, gás, tv, telefone, 
internet etc). Além de outros aspectos que merecem ser analisados para viabilizar sua implantação [1][3].

Segundo a concessionária de energia COPEL [8], muitos projetos são inviabilizados pela inexistência de um correto gerenciamento do uso do solo, de cadastro ou até do correto planejamento do empreendimento, antes mesmo da implantação das redes. É importante salientar que cada concessionária tem seu critério para o compartilhamento das obras civis e que é comum o compartilhamento de valas para a instalação de bancos de dutos, porém nenhuma delas permitem o compartilhamento de caixas de passagem e inspeção da rede elétrica de energia, com outras empresas prestadoras de serviços.

Independente da concessionária, a instalação de redes de distribuição subterrâneas de energia elétrica é uma opção mais onerosa quando comparado aos outros tipos de instalações e os principais custos das redes subterrâneas estão representados pelos transformadores, condutores isolados, conexões da rede primária e protetores de rede [3]. Nesse sentido, é imprescindível ter um levantamento preciso dos seus componentes na fase do planejamento como também a compatibilização do projeto com os demais sistemas.

Na última década, observa-se no Brasil e no mundo uma crescente adoção do uso do BIM e das suas ferremantas que em síntese permitem a construção virtual com informações de cada elemento utilizado. Tomando, como exemplo, um transformador, é possível adicionar a sua potência, tamanho, peso, tempo levado para instalação, fornecedores, valor entre outros dados. Em um segundo momento, é possível retirar as informações, de modo que elas possam ser utilizadas para: gerar lista de materiais, orçamentos, cronogramas, planejamentos etc [1]. Cabe destacar que o modelo mal detalhado ou mal elaborado, assim como o desconhecimento da maneira com a ferramenta de modelagem BIM gera as informações, podem comprometer esse processo de forma crítica [10][11][12].

Quadro 1: Softwares MEP BIM certificados (IFC $2 \times 3$ ) e aptos para modelagem de projetos de redes de distribuição subterrâneas de energia elétrica

\begin{tabular}{|l|l|l|l|}
\hline Fabricante & Software & Importa IFC & Exporta IFC \\
\hline ACCA Software & Edificus MEP & 2019 & Em andamento \\
\hline Autodesk-Revit & Autodesk Revit MEP & 2015 & 2013 \\
\hline Bentley Systems & AECOsim Building Designer & 2015 & 2015 \\
\hline CADMATIC & CADMATIC Elétrica e HVAC & Não tem & 2016 \\
\hline Data Design System & DDS-CAD MEP & Não tem & 2014 \\
\hline NEMETSCHEK Vectorworks & Vectorworks & 2013 & 2013 \\
\hline Progman & MagiCad & Não tem & 2016 \\
\hline VenturisIT GmbH & TRICAD MS & Em andamento & 2018 \\
\hline $\begin{array}{l}\text { Nota: Modificado pelos autores. } \\
\text { software/ }\end{array}$ & Fonte: https://www.buildingsmart.org/compliance/software-certification/certified-
\end{tabular}

A partir desta compreensão, realizou-se um levantamento a fim de saber quais os softwares BIM de modelagem poderiam ser utilizados na elaboração de projetos de redes de distribuição subterrâneas de energia elétrica e iluminação pública. Para tal fez-se uma busca no site da buildingSMART International e com isso foi possível identificar quais ferramentas eram certificadas para trabalhar na modelagem BIM. Em 
seguida, foram selecionados apenas os softwares que pudessem ser aplicados na confecção de modelos BIM de projetos desta natureza. Os resultados dessa ação são vistos no Quadro 1. Com base nesses resultados, optou-se em trabalhar como o Autodesk Revit, visto que é a ferramenta mais popular no território nacional.

\section{ESTUDO DE CASO}

Figura 2: Planta de implantação do Loteamento residencial - estudo de caso

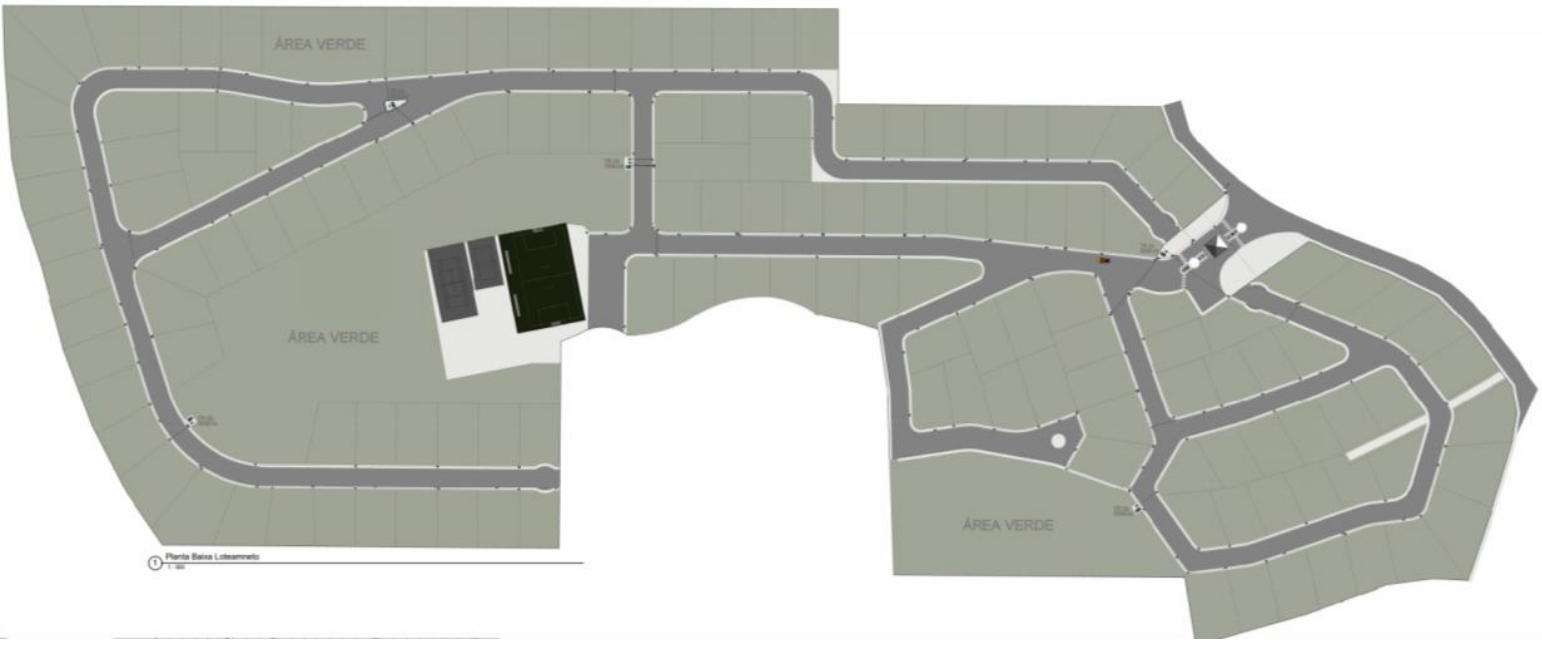

Fonte: os autores.

Figura 3: Perspectivas da rede de distribuição subterrânea de energia elétrica (Loteamento residencial - estudo de caso)
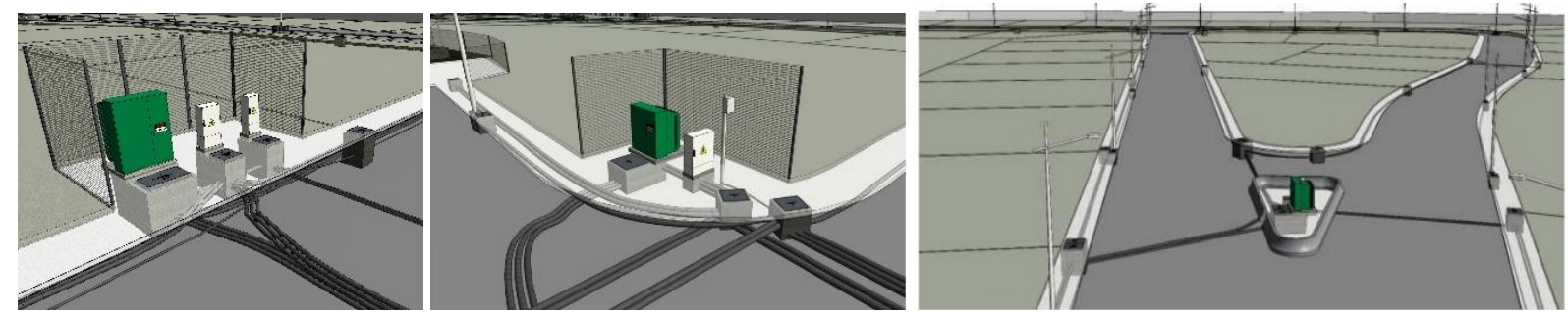

Fonte: os autores.

Figura 4: Perspectiva da rede de distribuição subterrânea de energia elétrica e outros sistemas (Loteamento residencial - estudo de caso)

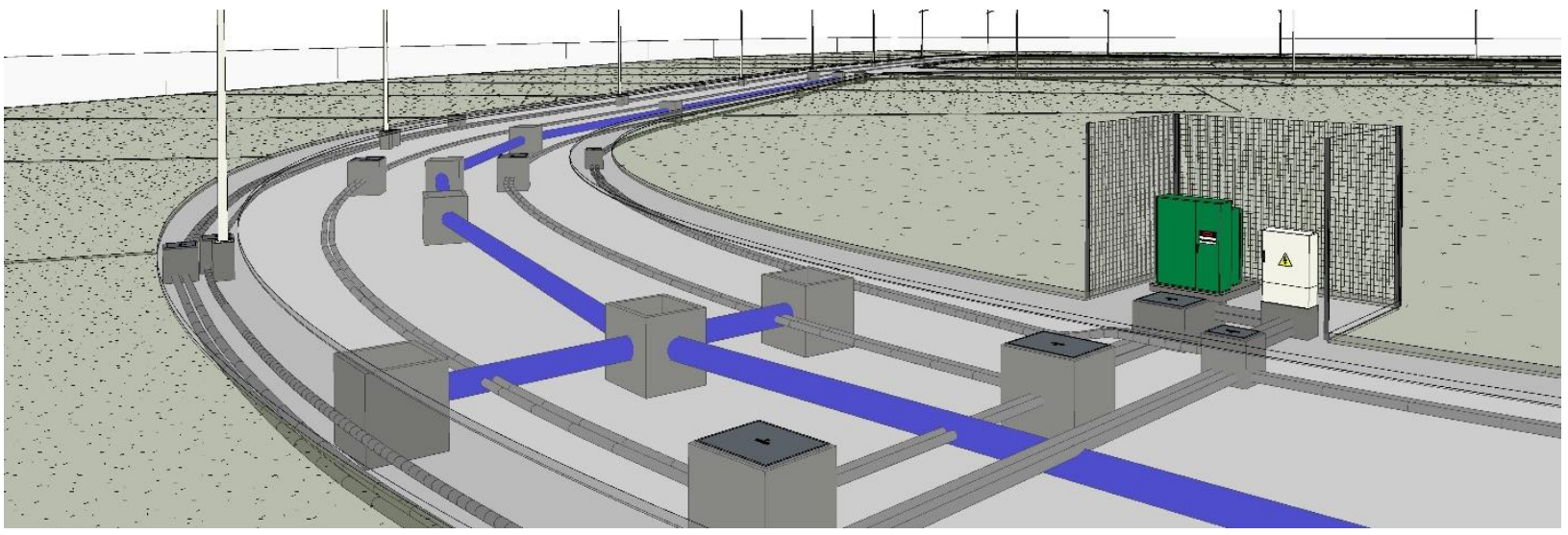

Fonte: os autores. 
Os projetos foram desenvolvidos com base em um loteamento residencial fictício, com área total aproximada de 148000 metros quadrados, composto de 142 lotes na faixa de 500 a 945 metros quadrados, além de uma área de lazer com aproximadamente 4800 metros quadrados e três espaços destinados a área verde, com aproximadamente 24200 metros quadrados (Figura 2).

Os projetos foram desenvolvidos utilizando o software Autodeks Revit o qual permitiu a geração de vistas das instalações em quaisquer posições de maneira simples e rápida, proporcionou uma melhor compreensão do projeto e extração de quantitativos (Figura 3 e 4) [1]. Foi adotado o LOD 300, onde os elementos são modelados a partir de informações como quantidade, tamanho, forma, localização e orientação segundo suas características reais, possibilitando deste modo a compatibilização entre os sistemas.

Paralelo a elaboração dos modelos BIM, foram confecionados memoriais descritivos e de cálculo dos projetos desenvolvidos a partir de critérios das normas das três concessionárias, CELESC D, CEMIG D e CPFL ENERGIA, de modo, a comparar as suas diferentes exigências. Os projetos contemplaram redes de distribuição subterrâneas de energia elétrica de média e baixa tensões, assim como, o dimensionamento de infraestrutura para rede de iluminação pública.

\section{RESULTADOS}

A partir do desenvolvimento dos projetos e modelos BIM, foram realizados os levantamentos de materiais, de equipamentos e de mão de obra necessários para a implantação da rede de distribuição subterrânea de energia elétrica e iluminação pública do loteamento. A obtenção do custo total estimado da obra é visto a seguir (Quadro 2).

Em síntese, observa-se uma variação entre os custos para a implantação da rede dependendo da região e da concessionária onde a obra será executada. Para a implantação da rede em regiões de concessão da CELESC $D$, temos um custo total de $\mathrm{R} \$ 2.484 .100,02$, já para implantação da mesma rede em regiões de concessão da CPFL ENERGIA, temos um custo total de $\mathrm{R} \$ 2.901 .311,55$ sendo este, $15,5 \%$ maior quando comparado ao valor da CELESC D. Na implantação da rede em regiões atendidas pela CEMIG D, temos um custo total de $\mathrm{R} \$ 3.188 .384,12$, sendo este $22,22 \%$ maior quando comparado ao valor da CELESC D e $10 \%$ maior que o custo da rede da CPFL ENERGIA. Apesar da variação nos custos totais da implantação da rede para as três concessionárias, verifica-se que os custos das obras eletromecânicas e civis para cada uma das três concessionarias se equiparam percentualmente quando comparados a seus valores totais (Quadro 3 ). 
Quadro 2: Custo total por concessionária

\begin{tabular}{|c|c|c|c|c|}
\hline \multirow[t]{2}{*}{ Tipo de obra } & \multirow[t]{2}{*}{ Itens } & \multicolumn{3}{|c|}{ Concessionária } \\
\hline & & CELESC D & CPFL ENERGIA & CEMIG D \\
\hline \multirow{3}{*}{ Obra civil } & Eletrodutos e acessórios & $\mathrm{R} \$ 146.470,21$ & $\mathrm{R} \$ 169.220,41$ & $\mathrm{R} \$ 194.113,21$ \\
\hline & Caixa e acessórios & $\mathrm{R} \$ 210.207,00$ & $\mathrm{R} \$ 316.898,00$ & $\mathrm{R} \$ 448.314,60$ \\
\hline & Mão de obra & $\mathrm{R} \$ 488.374,03$ & $\mathrm{R} \$ 562.062,72$ & $\mathrm{R} \$ 468.278,06$ \\
\hline \multirow{5}{*}{$\begin{array}{l}\text { Obra } \\
\text { eletromecânica }\end{array}$} & $\begin{array}{ll}\text { Transformadores } & \text { e } \\
\text { equipamentos de proteção } & \\
\end{array}$ & $\mathrm{R} \$ 329.284,30$ & $\mathrm{R} \$ 500.040,59$ & $\mathrm{R} \$ 601.747,24$ \\
\hline & Condutores e acessórios & $\mathrm{R} \$ 587.184,37$ & $\mathrm{R} \$ 548.133,45$ & $\mathrm{R} \$ 613.233,93$ \\
\hline & Iluminação pública & $\mathrm{R} \$ 303.304,50$ & $\mathrm{R} \$ 303.304,60$ & $\mathrm{R} \$ 348.922,60$ \\
\hline & Mão de obra & $\mathrm{R} \$ 417.276,51$ & $\mathrm{R} \$ 501.651,78$ & $\mathrm{R} \$ 513.774,48$ \\
\hline & Custo total (R\$) & $\mathrm{R} \$ 2.482 .100,92$ & $\mathrm{R} \$ 2.901 .311,55$ & $\mathrm{R} \$ 3.188 .384,12$ \\
\hline
\end{tabular}

Fonte: os autores.

Quadro 3: Comparativo Percentual das Obras Eletromecânicas x Civis

\begin{tabular}{|l|c|c|}
\hline Concessionárias & Obras Eletromecânicas & Obras Civis \\
\hline CELESC D & $66 \%$ & $34 \%$ \\
\hline CPFL ENERGIA & $65 \%$ & $35 \%$ \\
\hline CEMIG D & $65 \%$ & $35 \%$ \\
\hline
\end{tabular}

Fonte: os autores.

Quadro 4: Comparativo da Demanda Final por Concessionárias

\begin{tabular}{|l|c|}
\hline Concessionárias & Demanda Total (KVA) \\
\hline CELESC D & 1031 KVA \\
\hline CPFL ENERGIA & 947 KVA \\
\hline CEMIG D & 598 KVA \\
\hline
\end{tabular}

Fonte: os autores.

Quadro 5: Comparativo das Caixas e da Configuração de Rede

\begin{tabular}{|l|c|c|c|c|}
\hline Concessionárias & Rede Primária & Rede Secundária & Iuminação & Configuração \\
\hline CELESC D & Tipo D & Tipo B & Tipo A & Radial sem Recurso \\
\hline CPFL ENERGIA & Tipo CS-2 & Tipo CS-1 e CS-2 & Tipo CS-1 & Radial sem Recurso \\
\hline CEMIG D & Tpo ZD e VA & Tipo ZB & Tipo ZB & Anel Aberto \\
\hline
\end{tabular}

Fonte: os autores.

Quadro 6: Comparativo das Caixas e da Configuração de Rede

\begin{tabular}{|l|c|c|c|c|c|c|c|c|}
\hline Concessionárias & Qto & $\begin{array}{c}\text { Trafo 01 } \\
\text { (KVA) }\end{array}$ & $\begin{array}{c}\text { Trafo 02 } \\
\text { (KVA) }\end{array}$ & $\begin{array}{c}\text { Trafo 03 } \\
\text { (KVA) }\end{array}$ & $\begin{array}{c}\text { Trafo 04 } \\
\text { (KVA) }\end{array}$ & $\begin{array}{c}\text { Trafo 05 } \\
\text { (KVA) }\end{array}$ & $\begin{array}{c}\text { Trafo 06 } \\
\text { (KVA) }\end{array}$ & $\begin{array}{c}\text { Trafo 07 } \\
\text { (KVA) }\end{array}$ \\
\hline CELESC D & 5 & $300 \mathrm{kVA}$ & $300 \mathrm{kVA}$ & $300 \mathrm{kVA}$ & $300 \mathrm{kVA}$ & $300 \mathrm{kVA}$ & - & - \\
\hline CPFL ENERGIA & 5 & $300 \mathrm{kVA}$ & $300 \mathrm{kVA}$ & $300 \mathrm{kVA}$ & $300 \mathrm{kVA}$ & $300 \mathrm{kVA}$ & - & - \\
\hline CEMIG D & 7 & $150 \mathrm{kVA}$ & $75 \mathrm{kVA}$ & $150 \mathrm{kVA}$ & $75 \mathrm{kVA}$ & $75 \mathrm{kVA}$ & $150 \mathrm{kVA}$ & $150 \mathrm{kVA}$ \\
\hline
\end{tabular}

Fonte: os autores.

Quadro 7: Equipamentos de Proteção, Comando e Manobra

\begin{tabular}{|l|c|c|c|c|c|}
\hline Concessionárias & Para-ráios & $\begin{array}{c}\text { Chave } \\
\text { Fúsivel/Faca }\end{array}$ & $\begin{array}{c}\text { Chave } \\
\text { Seccionadora }\end{array}$ & Religador & Chave submersível \\
\hline CELESC D & Sim & Sim & Não & Não & Não \\
\hline CPFL ENERGIA & Sim & Não & Sim & Sim & Não \\
\hline CEMIG D & Sim & Sim & Não & Sim & Sim \\
\hline
\end{tabular}

Fonte: os autores. 
As variações dos custos finais para implantação da rede, se dá pelo fato de cada concessionária estabelecer valores e métodos diferentes para a obtenção da demanda mínima necessária por lote, gerando assim, uma grande variação entre as demandas totais previstas para o mesmo loteamento (Quadro 4).

Além da variação da demanda total de cada concessionária, outro fato que está diretamente ligado à variação dos custos finais da implantação da rede está nas obras civis (caixas e acessórios) e obras eletromecânicas (transformadores e equipamentos de proteção, comando e manobra), (Quadro 2). A variação dos custos referentes as caixas e acessórios estão relacionados ao tipo de configuração da rede utilizada e das dimensões das caixas.

A CELESC D, utiliza configuração de rede radial sem recurso para a rede primária, possuindo uma infraestrutura compacta, e utiliza caixas do tipo B nos trechos que só possuem rede secundaria (Quadro 5), gerando um custo para essas infraestruturas de $\mathrm{R} \$ 210.207,00$. A CPFL ENERGIA, utiliza configuração de rede radial com recurso para a rede primária, com infraestrutura semelhante à da CELESC D, porém utiliza caixas do tipo CS-2 (Quadro 5), com dimensões maiores quando comparadas às caixas utilizadas pela CELESC D, nos pontos de derivação e de instalação de BMI-CPFL, gerando um custo de $R \$ 316.898,00$, sendo este $50,75 \%$ maior, quando comparado os custos da CELESC D.

Por sua vez a CEMIG D, utiliza configuração de rede anel aberto para a rede primária, exigindo uma infraestrutura maior e consequentemente um número superior de caixas quando comparada as demais concessionárias, sendo ainda, essa infraestrutura separada da rede de baixa tenção (Quadro 5), essas diferenças geram um custo de $\mathrm{R} \$ 448.314,60$, sendo estes custos $113,27 \%$ maior que custos da CELESC D e $41,47 \%$ maior que os custos da CPFL ENERGIA.

A CELESCD, apesar de apresentar a maior demanda total prevista, necessita de apenas cinco transformadores para atendimento da demanda total, sendo suas potências e quantidades expressas no Quadro 6. Ainda, para a demanda total, a concessionária exige a instalação, no poste de transição, de chave fusível e pára-raios para a proteção, comando e manobra da rede (Quadro 7), gerando um custo para essas infraestruturas de $R \$ 329.284,30$. A CPFL ENERGIA, apresenta demanda total semelhante à da CELESC $D$ (Quadro 3) e quantidade de transformadores iguais, porém com potências diferentes (Quadro 6).

A CEMIG D, apesar de possuir a menor demanda total entre as três concessionárias, possui transformadores padronizados com menor potência quando comparados à CELESC D e CPFL ENERGIA, necessitando assim de uma quantidade maior para o atendimento da demanda total (Quadro 6). Para a proteção, comando e manobra da rede, a CEMIG D, exige, no poste de transição, além da instalação de pára-raios e de religador automático, a instalação de chave faca, (Quadro 7) e, ainda, chave de média tensão submersível de três vias instalada logo após o poste de transição, (Quadro 7). Essas diferenças geram um custo de $R \$ 601.747,24$, sendo estes custos $82,74 \%$ maior que custos da CELESC D e $20,33 \%$ maior que os custos da CPFL ENERGIA. 
Por fim, os custos podem ser determinantes para uma possível tomada de decisão na escolha do local para futuros empreendimentos por parte de investidores. Dentre as concessionárias estudadas, a CPFL ENERGIA destaca-se no quesito de confiabilidade, já que a mesma possui sua configuração de rede radial com recursos, que dificulta a interrupção do fornecimento de energia elétrica aos clientes.

A CEMIG D possui configuração de rede anel aberto, que possibilita recursos internos. No entanto, tal segurança representa aumento significativo nas infraestruturas, caracterizando assim um custo elevado quando comparado às outras duas concessionárias. A CELESC D, apresenta uma configuração de rede radial sem recurso, sujeita a interrupções, porém mais compacta. No entanto, para cargas prioritárias, utiliza configurações de rede radial com recurso. Ou seja, a depender da concessionária na qual o projeto será implantado, as configurações das redes serão sempre diferentes e, consequentemente, seus custos.

\section{CONCLUSÃO}

As análises produzidas explicitam tanto a grande diferença em termos do que cada concessionária entrega no que se refere à Demanda Total, e também na solução diferenciada que cada uma propõe. O estudo mostrou-se complexo, pois, apesar de cada concessionária possuir uma normativa para projetos de redes de distribuição subterrânea de energia elétrica em loteamento, estas direcionavam para uma extensa quantidade de outras normas e especificações técnicas complementares, algumas dessas de difícil acesso.

Cabe destacar que as disparidades entre as concessionárias implicam em custos distintos e que independem do uso ou não do BIM. Por outro lado, a ferramenta de autoria BIM utilizada facilitou o processo de comparação, visto que permitiu a extração de dados de modo mais rápido e mais preciso quando contraposto ao processo tradicional. Além de ter proporpocionado a visualização dos detalhes do projeto através de seu modelo 3D, a geração de vistas em quaisquer posições, que por sua vez permitiu uma melhor compreensão do projeto e de alguns critérios estabelecidos pelas concessionárias, e ainda a compatibilização com os demais sistemas. Em síntese, a ferramenta escolhida possibilitou os seguientes usos do modelo[13]: Documentação 2D; Detalhamento 3D; Modelagem do Terreno; Design Autoral; Detecção de Interferências; Análise de construtibilidade; Extração de Quantitativos.

Por fim, a pesquisa respondeu o objetivo geral que foi desenvolver um comparativo entre três concessionárias (CELESC D, CEMIG D e CPFL ENERGIA) a partir de modelos BIM. E respondeu de que maneira o uso de uma ferramenta de autoria BIM pode contribuir na geração de projetos de distribuição subterrânea de energia elétrica.

\section{AGRADECIMENTOS}

À Quatre Ensino Especializado pelo apoio à pesquisa. 


\section{REFERÊNCIAS}

[1] INÁCIO, E. C, GOMES, W.M. Redes de distribuição subterrâneas de energia elétrica: comparativo entre os requisitos das concessionárias CELES D, CPFL ENERGIA E CEMIG D utilizando modelos BIM. 2018. $167 \mathrm{f}$. Trabalho de conclusão de curso (Bacharelado em Engenharia Elétrica) - Universidade do Sul de Santa Catarina, Palhoça, 2018

[2] FERNANDES, P. J. Sistema de distribuição subterrânea de energia elétrica. 2018. 83 f. Trabalho de conclusão de curso (Bacharelado em Engenharia Elétrica) - Centro Universitário Augusto Motta (UNISUAM), Rio de Janeiro, 2018.

[3] AZEVEDO, F. A. Otimização de rede de distribuição de energia elétrica subterrânea reticulada através de algoritmos genéticos. 2010. 138 f. Dissertação (Mestrado em Engenharia Elétrica) - Universidade Federal do Paraná, Curitiba, 2010.

[4] CHECCUCCI, Érica de S. Teses e dissertações brasileiras sobre BIM: uma análise do período de 2013 a 2018. PARC Pesquisa em Arquitetura e Construção, Campinas, v. 10, p. e019008, 2019. DOI: 10.20396/parc.v10i0.8653708. Disponível em:

https://periodicos.sbu.unicamp.br/ojs/index.php/parc/article/view/8653708. Acesso em: 6 mar. 2021

[5] BIM DICTIONARY. Building Information Modelling (BIM). BIM Dictionary. Disponível em: $<$ https://bimdictionary.com/en/building-information-modelling/1/>. Acesso em: 2 mar. 2021.

[6] ASSOCIAÇÃO BRASILEIRA DE NORMAS TÉCNICAS. NBR 5410: Instalações elétricas de baixa tensão. Rio de Janeiro, 2004.

[7] ASSOCIAÇÃO BRASILEIRA DE NORMAS TÉCNICAS. NBR 14039: Instalações elétricas de média tensão. Rio de Janeiro, 200.

[8] COPEL. Padrões Construtivos Rede de Distribuição Subterrânea. COPEL. Disponível em: < http://www.copel.com/hpcopel/redesub/padroes_construtivos.html>. Acesso em: 27 de agosto de 2017.

[9] CHAGAS G.F.C. Análise comparativa entre fluxos de modelagem para orçamentação em BIM a partir da aplicação em uma residência multifamiliar situada no município de Natal/RN. 2019. 77f. Trabalho de conclusão de curso (Bacharelado em Engenharia Civil) - Universidade Federal do Rio Grande do Norte, Natal, 2019.

[10] GIESTA J. P. et al. O uso da tecnologia BIM na quantificação e no planejamento: estudo de caso de residência RN- BR. In: CONGRESSO PORTUGUÊS DE BUILDING INFORMATION MODELLING, 2., 2018, Anais [...] Lisboa, Faculdade de Engenharia, Universidade de Lisboa, 2018, pp. 487-495. DOI: https://doi.org/10.5281/zenodo.1226766

[11] TRINDADE. L. D.; SANTOS.E. T. Definição de diretrizes de modelagem BIM para quantificação em diferentes etapas do processo de projeto. In: Simpósio Brasileiro de Tecnologia da Informação e Comunicação na Construção e Simpósio Brasileiro de Gestão e Economia da Construção, 2017, Anais [...]. Universidade Federal do Ceará, Fortaleza, 2017.

[12] SUCCAR, B., SALEEB, N., SHER, W. Model Uses: Foundations for Modular Requirements Clarification Language. In: Australasian Universities Building Education (AUBEA2016), 2016, Cairns, Australia. Proceedings [...]. Cairns, 2016, p. 45 - 57. Disponível em: <https://www.researchgate.net/publication/303013287_Model_Uses_Foundations_for_ a_Modular_Requirements_Clarification_Language>. Acesso em: 29 de abril de 2021. 